\title{
Quantifying social preference in young children using two tasks on a mobile platform
}

Authors: Indu Dubey ${ }^{1, \# a}$, Simon Brett ${ }^{1}$, Liliana Ruta ${ }^{2}$, Rahul Bishain ${ }^{3}$, Sharat Chandran ${ }^{3}$, Supriya Bhavnani ${ }^{4,10}$, Matthew K. Belmonte ${ }^{5,6}$, Georgia Lockwood Estrin ${ }^{7}$, Mark Johnson ${ }^{8}$, Teodora Gliga ${ }^{9}$, and Bhismadev Chakrabarti ${ }^{1 *}$, on behalf of the START consortium ${ }^{\wedge}$

Affiliations: (1) Centre for Autism, School of Psychology \& Clinical Language Sciences, University of Reading, Reading, United Kingdom, (2) Institute of Applied Sciences and Intelligent Systems, National Research Council of Italy (CNR), Messina, Italy, (3) Department of Computer Science and Engineering, Indian Institute of Technology Bombay, Mumbai, India, (4) Centre for Chronic Conditions and Injuries, Public Health Foundation of India, Gurgaon, India, (5) Com DEALL Trust, Bangalore, India, (6) Division of Psychology, Nottingham Trent University, Nottingham, United Kingdom, (7) Centre for Brain and Cognitive Development, Birkbeck University of London, London, United Kingdom, (8) School of Psychology, University of Cambridge, Cambridge, United Kingdom, (9) School of Psychology, University of East Anglia, Norwich, United Kingdom, (10) Sangath, New Delhi, India.

\#a Current address: Division of Psychology, De Montfort University, Leicester, United Kingdom

\section{* Corresponding Author}

Email: b.chakrabarti@reading.ac.uk (BC)

${ }^{\wedge}$ Membership of the START consortium is provided in the Acknowledgements. 


\begin{abstract}
Children typically prefer social stimuli (e.g. faces, smiles) over non-social stimuli (e.g. natural scene, household objects). This social preference is believed to be an essential building block for later social skills and healthy social development. Measuring social reward responsiveness poses an empirical challenge, as it encompasses multiple underlying processes. In this study, we use a preferential looking task and an instrumental choice task to capture different potential processes underlying social preference, in over 100 typically developing 3-9 year old children. Children spent longer looking at social stimuli in the preferential looking task but did not show a similar preference for social rewards on the instrumental choice task. This study highlights the importance of choice of paradigms when evaluating social preference and their potential impact on understanding social reward responsivity.
\end{abstract}

Key words: eye tracking, choice task, social reward, social motivation, digital health 


\section{Introduction}

Social interactions are one of the core components of our daily lives. Neurotypical infants make attempts to engage in social interaction from as early as 6-8 weeks of age, responding to their caregivers by smiling or cooing [1]. Children spend longer looking at faces than at comparable non-social stimuli with matched spatial frequency [2], they prefer biological to non-biological motion [3], and smile in response to social but not non-social stimuli [4]. These studies indicate high social reward responsivity in early years of life.

Paradigms measuring social reward responsivity can broadly be categorised into two classes. One class consists of passive paradigms that present the social stimulus (e.g. a smiling face) and measure the pleasure/consummatory aspect of the participant's response. These measures can be observational (e.g. gaze duration in an eye-tracking measure, or rewardrelated neural response in a neuroimaging measure), or self-reported (e.g. 'rate how much you like this'). In contrast, the second class of paradigms are instrumental tasks that present conventionally rewarding social stimuli contingent on the performance of a particular behaviour (typically a motor act such as pressing buttons). In these tasks, the choice behaviour as indexed through the number or speed of the responses is taken to provide a measure of social reward responsivity.

While self-report studies arguably provide a subjective account of how rewarding a stimulus is, ratings and language-based tools are easily influenced by experimenter or subjective biases and are not suitable for use in populations with low verbal abilities, such as very young children [5-7]. To minimise such biases, preferential looking paradigms using camera-based or continuous eye-tracking technology have been used to index relative reward value $[8,9]$. These studies are based on the assumption that longer gaze duration toward a specific set of stimuli indicates greater interest and greater positive value for those stimuli [911]. In a typical preferential looking paradigm for social versus non-social rewards, two competing stimuli- one of each type- are simultaneously presented while participants' gaze is 
tracked $[12,13]$. These studies have demonstrated that neurotypical children and adults gaze longer at social than non-social stimuli [13-16]. Children as well as adults thus demonstrate a robust preference for social rewards, when measured using visual preference paradigms.

Comparatively fewer studies have used an instrumental task to test preference for social stimuli. One of the early attempts at addressing this gap was the Social Incentive Delay task (SID), in which participants are aware of the reward (social/non-social) they would receive at the end of each trial on a reaction time game [17]. Here, reaction time in anticipation of social reward is used as a measure of social reward responsivity. Neurotypical adults react faster reaction time for monetary rewards compared to social (smiling faces) rewards [17]. Another study using a similar paradigm compared two age groups of younger adults (20-28 years) and older adults (60-78 years), both of whose hit rates (accuracies), were significantly higher for monetary than social (smiling faces) rewards $[17,18]$. The same study found that the reaction time was influenced by the reward magnitude and not the type (social vs. monetary). The second set of studies examining social reward responsivity in instrumental paradigms used effort as an index of social reward responsivity. In tasks of this type, participants exert different amounts of effort (made through complex or multiple button presses) to obtain a social or a nonsocial reward $[19,20]$. These tasks demonstrate that neurotypical adults carefully evaluate the level of effort required and available outcomes while making a choice. They invest more effort to get social reward than non-social but no such preference is seen in neurotypical adolescents [20-22]. Ruta and colleagues used a similar but simpler paradigm targeted for younger children (14-68 months), where children choose one of two buttons to look at social or non-social images [23]. They found that neurotypical children do not show any preference for the 'social' stimulus in this 'button' task.

In summary, studies evaluating social reward responsivity in the neurotypical population have reported mixed findings. On one hand, in paradigms where the stimuli are processed passively or with minimum effort, participants typically show a higher reward-related response to 
social stimuli. On the other hand, paradigms that involve an instrumental component do not always show this 'social advantage'. The scope of inference has been limited by the absence of any study using both of these paradigms in the same set of individuals. The current study addresses this gap using both a preferential looking task and an instrumental choice task in young children administered on a mobile platform (a tablet PC). In line with previous lab-based studies, we hypothesise that children will show a visual preference for social stimuli in the passive viewing paradigm, and that a similar social preference will not be noted in the instrumental choice task.

\section{Methods}

Participants: Children between ages 3-9 years were recruited from the summer scientist week - a public science engagement event at the University of Nottingham, where parents from the local community brought in their children. Children took part in a number of experiments, each of which was conducted in a small booth within a large open space. The study used a convenience sampling method, and accordingly sample demographics e.g. age range, gender ratio, and number of participants, were influenced by the nature of the event. The preferential looking task (described in the tools section) was completed by 101 children ( 57 females). Their mean age was 5.69 years $(S D \pm 1.38)$. The button task (described in the tools section) was completed by 105 children ( 52 females). Their mean age was 5.64 years (SD \pm 1.60$)$. Over $50 \%$ $(n=52)$ of these participants (31 females; mean age 5.58 SD \pm 1.23$)$ completed both the tasks.

Stimuli: The social and non-social video stimuli used in the tasks were taken from the commercial image/video sharing website www.shutterstock.com. The social stimuli included videos of one or more children smiling and looking at the camera or a parent and child smiling and interacting with each other. The non-social stimuli included videos of falling pieces of puzzles, dynamic patterns, spinning wind-fans or washing machines. Each video clip (no 
sounds) was trimmed to 5 seconds for both the tasks. Both tasks were presented on a tablet PC (Samsung 10.1 tablet SM P600) running the START app https://startproject.bhismalab.org[24].

\section{Measures}

Preferential looking task: This task was similar to paradigms used by Pierce and colleagues to measure social preference in children on the autism spectrum $[13,25]$ (we use the phrases children/individuals on the autism spectrum and autistic children/individuals interchangeably to acknowledge the diversity of views regarding terminology within the autism community). Four pairs of social and non-social videos (social videos showing children smiling, and non-social videos showing spinning fans or washing machines) each presented twice in a fixed order. The side of presentation and video type (social/non-social) was counterbalanced (Fig 1). Each video covered nearly half of the screen, leaving a gap of $0.5 \mathrm{~cm}$ between them.

Button task: This task was based on a similar tablet-based task used previously to measure social preference in young children on the autism spectrum and matched control [23]. This was an instrumental choice task in which participants were shown two "buttons" on the screen, which presented either a social or a non-social stimulus when touched. The position of the "buttons" on the screen was randomly changed in every trial to overcome any side or handedness bias. Unlike the original version of the task which used static pictures, the stimuli in this version were two sets of videos: 1) social e.g. a child swimming underwater and waving or a mother and a child hugging each other, and 2) non-social e.g. puzzle pieces falling in place or a geometric wave-like pattern. Videos instead of images were used to increase salience [26]. These videos were presented in full screen display after the participant touched the button to make the choice. The association between buttons and the linked set of stimuli was explicitly demonstrated to the children before starting the task. There were eight choice trials, for which the responses were captured using the device's touch sensors.

British picture vocabulary scale (BPVS) $3^{\text {rd }}$ edition: Verbal intelligence was measured using 
the British Picture Vocabulary Scale (BPVS) $3^{\text {rd }}$ edition [27]. This measure allowed us to test the impact of intelligence on the putative metrics of social preference. We report the raw score on BPVS for statistical analysis.

Social aptitude scale (SAS): The Social Aptitude Scale (SAS) is a 10-item parent report scale to evaluate social skills in children (5-16 years) [28]. This measure allows for a potential test of criterion validity in view of the suggested link between social motivation and social skills $[29,30]$.

Procedure: Ethical approval for the study was provided by the ethics committee of the School of Psychology, University of Nottingham. All procedures performed in this study were in accordance with the ethical standards of the University of Nottingham and with the 1964 Helsinki declaration and its later amendment in 2008. Informed consent was obtained from the primary caretaker of all individual participants (under 18 years of age). Each participant provided verbal assent prior to taking part in the study. Participants were invited to play some "games" on a tablet in a quiet section of a large room (shared with other researchers). They sat at a childsized table and chair and completed both tasks in a single sitting. It took less than 10 minutes for each child to complete both the tasks. During the public engagement event, participants were also evaluated for their verbal intelligence using BPVS and their primary caretakers completed the SAS. These scores were made available to researchers after data collection. All the children (irrespective of their performance) were given a gift pack for taking part in the public engagement event.

Preferential looking task: The tablet was positioned upright on a stand facing the child (see Fig 1a). The task started with a preparatory phase during which tablet's position was adjusted to ensure a) the child's eyes and face were visible to the front camera of the tablet, b) there was sufficient illumination, c) the face was within $35-50 \mathrm{~cm}$ from the tablet, and d) the tablet was stable. On each trial a set of two video clips: a social and non-social were shown on the screen for five seconds, examples shown in Fig $1 \mathrm{~b}$ and a video file available in S1 appendix. The eight 
trials continued without any gap or central fixation cue and the participants were encouraged to stay still while looking at the screen.

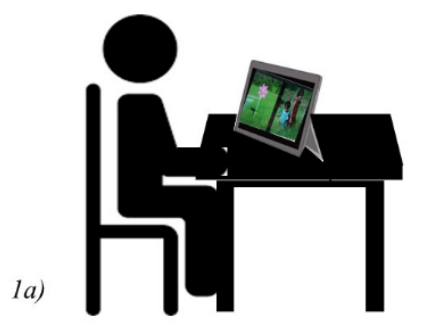

1b)

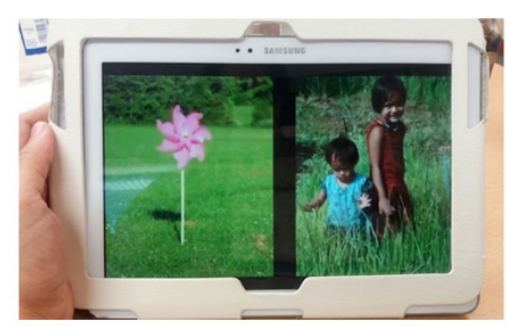

Fig 1a) Schematic presentation of the setup, 1b) A sample trial from the preferential looking task shown on a tablet device.

Button task: The task was presented with the tablet placed flat on the table. It began with a demonstration phase showing the two 'buttons' present at the beginning of every trial. The experimenter then verbally instructed and demonstrated to the children that one of these buttons had videos of 'people' while the other had the videos of 'things' (see example Fig 2 and sample video of the task in S1 appendix). Participants were then given a chance to touch the two buttons and observe examples of stimuli. They were then shown eight experimental trials in which they were free to choose to touch any button to look at the social or non-social videos.

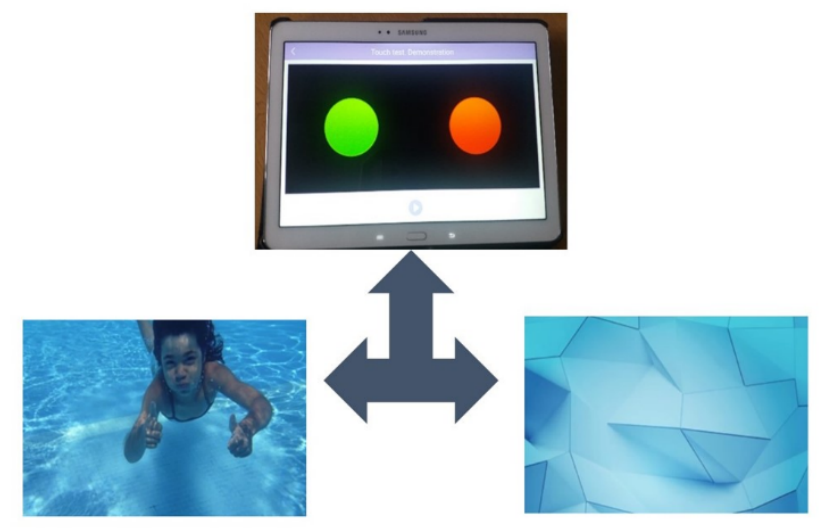


Fig 2: A sample trial from button task showing choice presented on the tablet screen and linked sample of videos.

\section{Data processing}

Preferential looking task: The face and eyes from the video recording using the front camera of the tablet were automatically extracted while the participants looked at the screen during the task. This video feed was then processed using an adapted version of a gaze estimation algorithm [31,32]. The algorithm utilizes a deep neural network model trained on the gazecapture database. The database is a corpus of 2.5 million images collated from 1474 subjects. The original model is shown to have achieved state of the art results with $2.53 \mathrm{~cm}$ prediction error on iOS-based tablet devices. We observed that the model was not trained on children of the requisite age group, and neither have the experiments been conducted in a casual setting on Android-based devices. We therefore re-validated our implementation of the algorithm on data collected from children performing the preferential looking task by manually annotating 4952 frames extracted from the videos of 8 children. We observed a $91.23 \%$ accuracy in validation using this approach. Here, accuracy refers to the percentage of frames correctly labeled by the algorithm as compared to the manual labels. For the statistical test reported below, the percentage of gaze on social stimuli was calculated as follows:

Percentage of gaze on social stimuli= $\{\mathrm{N}$ (frames with gaze on social stimuli)/N(total frames with gaze on social or non-social stimuli) $\}^{*} 100$

Button task: Proportion of trials on which participant chose the button linked with the social stimulus was calculated for each participant as an index of social preference.

Social preference=

$\{\mathrm{N} \text { (trials on which social stimulus was chosen)/N(total trials completed) }\}^{*} 100$ 
Statistical analyses: Participants' age, gender, verbal intelligence, and social skills were included in all analyses reported. A one-sample $t$ test against a chance value of $50 \%$ was run to ascertain any significant preference for social stimuli on both the tasks. A general linear model was run with gender and age as predictors of proportional looking to social stimuli in the preferential looking task and social preference on button task. The interrelationship between social preference as measured on the two tasks was tested using a correlation analysis. Partial correlations, controlling for the effects of age and gender, were run to explore the relation between each measure of social preference with verbal intelligence and social skills separately.

\section{Results}

An outlier check was done for both sets of data using Cook's distance (age as independent variable) taking cut off as $4 / n$. This identified seven potential outliers for the preferential looking task and nine potential outliers for the button task. Statistical analyses were run without these participants did not change the findings, so we report the results with the larger sample including all the participants.

Preferential looking task: The algorithm was successful in detecting the gaze location on mean $99 \% \pm 4.43$ of frames. The results from this task suggest that participants looked towards the social stimuli more than expected by chance $(t(100)=12.92, p<.001, M=63.56$, $S D=10.55)$. There was a significant main effect of $\operatorname{gender}(F(1,97)=8.37, p=.005, \beta=25.91)$, female participants demonstrating a stronger preference for social stimuli $(M=65.18, S D=8.90)$ than male participants $(M=61.47, S D=12.15)$. There is also a significant interaction between age and gender $(F(1,97)=6.52, p=.01, \beta=-3.96)$. To understand these results better we visualised the social preference of male and female participants over different age groups (the age data are presented in categories in Fig 3 only for visualisation). The main effect of gender might be driven primarily by younger females who have higher preference for social stimuli then 
younger males, see Fig 3 . There was no significant main effect of age $(F(1,97)=.65, p=.421$, $\beta=2.61$ ). There was no significant correlation between the proportion of gaze on social stimuli and verbal intelligence (BPVS raw score) $\left(r_{\mathrm{s}}(86)=.099, p=.358\right)$ controlling for the effects of age and gender. Since SAS is developed for 5-16 years of age, we calculated a partial correlation (age and gender) between the proportion of gaze on social stimuli and social skills as evaluated on SAS for only children age 5 years or above. The correlation between the two constructs falls below the traditional thresholds of statistical significance $(r(67)=.214, p=$ $.077)$.

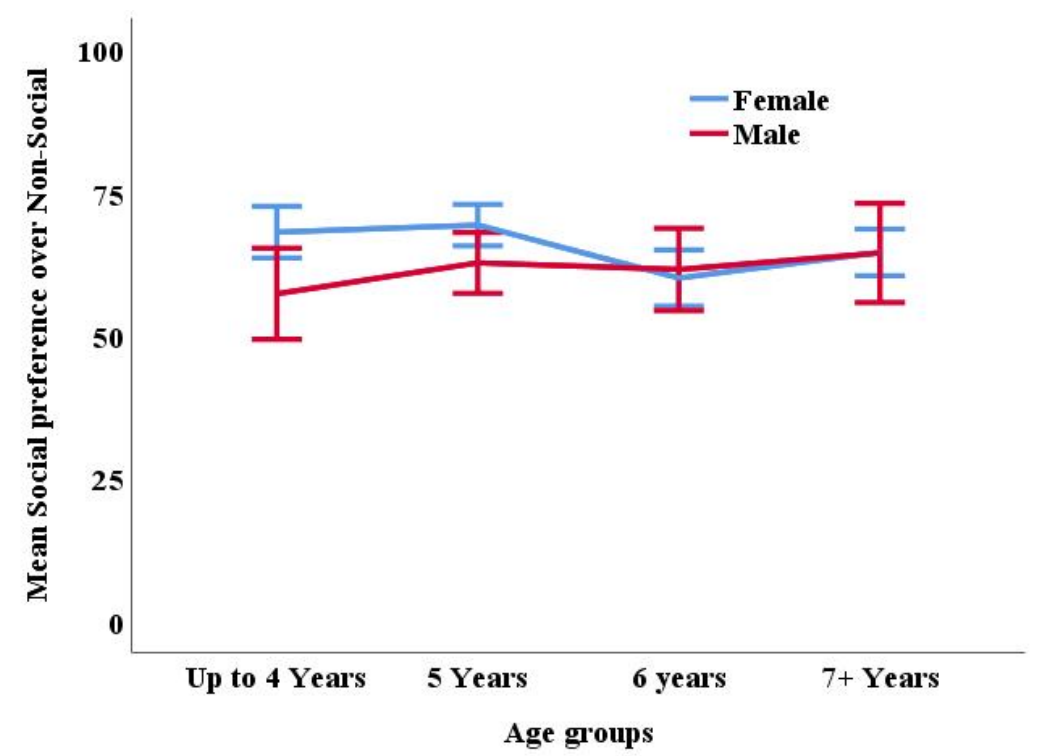

Fig 3: Line graph shows the interaction between age and gender when predicting social preference on preferential looking task.

Button task: Participants did not choose the button for social stimuli more than expected by chance $(t(104)=.540, p=.590, M=50.83, S D=15.82)$. No significant effects of age $(F(1,101)=$ 1.132, $p=.290, \beta=-1.21)$, gender $(F(1,101)=0.022, p=.881, \beta=1.72)$ or their interaction $(F$ $(1,101)=0.025, p=.874, \beta=.313)$ were noted. There was no significant correlation between the social preference and verbal intelligence (BPVS raw) $\left(r_{\mathrm{s}}(86)=-.056, p=.603\right.$ ) after partialling out the effects of age and gender. Social preference and social skills in children more 
than 5 years of age were significantly correlated $(r(67)=.317, p=.008)$ while partialling out effects of age and gender (Fig 4).

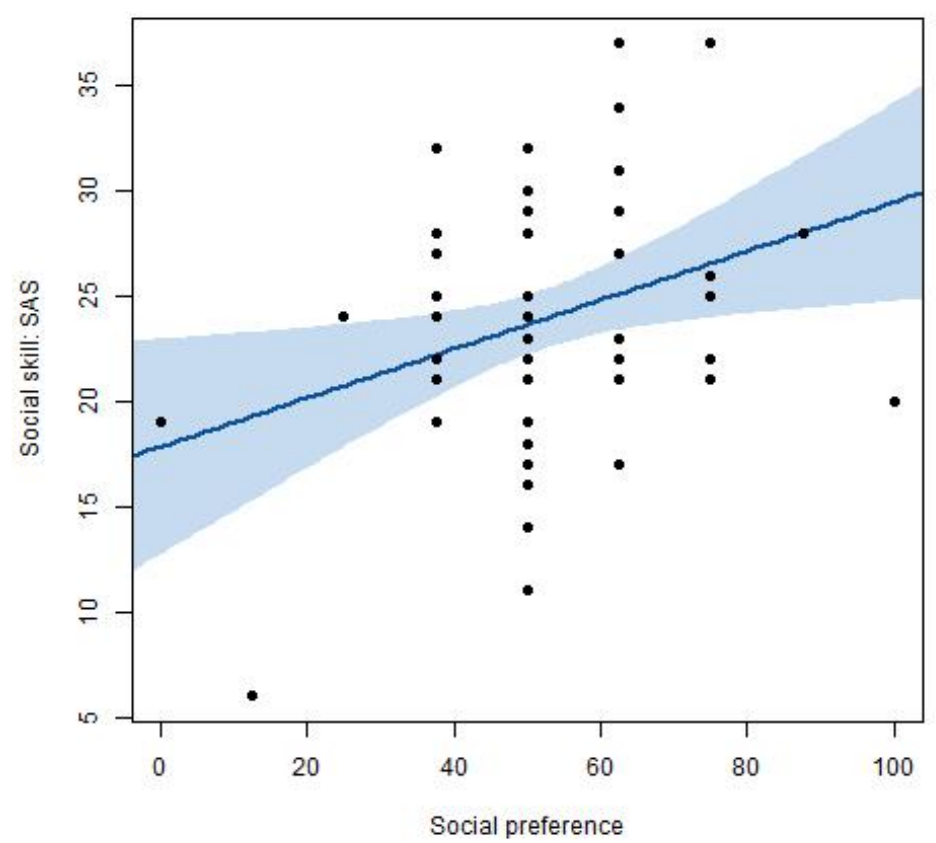

Fig 4: Scatter graph for social skills as measured on SAS and the social preference as measured on button task (for participants above age 5 years).

Inter-task comparison: Social preferences across the two tasks: Preferential looking and Button tasks (completed by 52 participants) were not correlated with each other $\left(r_{\mathrm{s}}(50)=.136, p\right.$ $=.338)$.

\section{Discussion}

This study evaluated two putative task measures of social reward responsivity in typically developing children using a tablet PC. We found a preference for social over non-social stimuli in a preferential looking task, replicating results from similar studies using lab-based eyetrackers $[14,15]$. This preference for social stimuli was not seen in the instrumental choice 
(button) task. Despite this absence of overall social preference in the button task, a positive relationship was noted between children's social skills and their preference to choose the button associated with the social stimulus, replicating previous results. Interestingly, these two measures of social preference were uncorrelated in this dataset, pointing to the possibility that these tasks index distinct processes within the broader construct of social reward responsivity. We discuss these results below, highlighting the importance of task choice in measuring social reward responsivity and its potential impact.

Studies evaluating visual preference for social stimuli in neurotypical children suggest that they spend longer looking at social stimuli $[15,16,25,33,34]$. It is also suggested that the more complex social stimuli (depicting multiple individuals), when used in a preferential looking task, are likely to elicit greater social preference $[35,36]$. In the present study, we report similar results showing social preference in neurotypical children using a tablet-based preferential looking task. A significant gender difference was noted, with females showing greater preferential attention to social over non-social stimuli. Higher preference for social stimuli in females has been reported previously at both behavioural and neural levels suggesting a sex-linked innate bias in social preference $[37,38]$. Interestingly, this differential preference for social stimuli in females was more prominent in the younger participants.

Unlike preferential looking measures, behavioural approach or instrumental choice tasks have been less consistent in indicating preference for social stimuli in neurotypical children [2023]. In the present study, we replicated the findings by Ruta and colleagues [23] using an adapted version of the original task. Children showed no significant preference for social stimuli on the button task. Interestingly however, a reduced preference for pressing the button associated with the social stimulus was associated with low scores on a measure of social skills (SAS). Responses to social rewards have been suggested to be linked to social skills $[29,30]$. The results from the button task are consistent with the original report by Ruta and colleagues [23], where typically developing children did not show an overall social preference. Even though 
the button task is a simpler version of the instrumental tasks used previously, it still requires participants to remember the association between the cue (button) and reward (linked videos). Furthermore, this task's requirment to make motor movements to seek the social reward imposes additional demands. Preferential looking tasks, on the other hand, impose no such demands and hence might quantify "automatic" or hedonic preference for social stimuli. This also suggests that the two paradigms used in this study could correspond roughly to the theoretical constructs of 'liking' and 'wanting' within reward processing [39]. In this account, liking is defined as the conscious experience of pleasure or the hedonic value of the rewarding stimulus, and wanting as the incentive salience that triggers approach behaviour towards the rewarding stimulus. While behavioural response of making effort to seek a stimulus, as in our button task, can be seen as an index of wanting, consumption of the stimulus leading to an experience of instant hedonic pleasure, as in the preferential looking task, can be an index of liking [40]. It has also been demonstrated previously that longer visual fixations might indicate higher reward value of a stimulus [9,41]. Hence, while both of our paradigms measure reward responsivity, preferential looking might give a better estimate of social liking (consummatory behaviour), while button-pressing might give a better estimate of social wanting (seeking and anticipatory behaviour) [42]. This pair of complementary constructs carries implications for understanding differences in reward responsivity in typical and atypical populations.

Importantly, both the preferential looking as well as the button task for measuring social reward responsivity were administered on an overlapping (50\%) sample of children, making it possible to evaluate the divergent patterns of normative data on these two experimental measures and to test their inter-relationship. Dissociations in different aspects of reward processing have been reported for stimuli such as food or drugs, and these have been linked with atypical behaviour $[43,44]$. Questionnaire measures have pointed toward potential dissociation between reward processing components, as well as between self-report and behavioural measures of social reward responsivity in clinical population $[45,46]$. Accounts of 
reduced social reward responsivity in clinical population may therefore benefit from a more rigorous assessment of distinct components of reward processing in future studies.

Several limitations arise from the constraint of implementing these tasks in a new, mobile computing environment. First, the order of presentation of stimuli in the preferential looking task was non-random (social stimuli on the left in the first four trials and on the right in the next four trials). While this fixed order of counterbalanced stimuli should not influence the inferences drawn, we have fully randomised the order of presentation in a later study. Second, in the instrumental task the association between buttons and stimulus type (social/non-social) was not counterbalanced across participants. We have added this feature in the later version of the task, to guard against confounds with colour preferences (e.g. if some children do not like the colour orange, and will never press an orange button). While we aimed to administer both the preferential looking and the button task on all the participants, technical and procedural constraints made this possible only for a part (50\%) of the sample. However, we find no reason to expect any of these limitations to change the interpretation.

\section{Conclusion}

The present study reports data from two tasks that evaluate two complementary aspects of social reward responsivity, in young children. The results highlight the importance of using a range of measures to tap these different components of social reward processing. These complementary measures of social reward responsivity can provide a theoretically informed approach to stratification within and across neurodevelopmental conditions.

\section{Acknowledgment}

Data presented in this paper has been presented as a preprint at PsyArXiv [47]. 
START Consortium members are listed here in alphabetical order: Matthew Belmonte ${ }^{1,2}$, Supriya Bhavnani ${ }^{3,4}$, Rahul Bishain ${ }^{5}$, Bhismadev Chakrabarti ${ }^{6}$, Sharat Chandran ${ }^{5}$, Jayshree Dasgupta $^{3,4}$, Gauri Divan ${ }^{4}$, Indu Dubey ${ }^{6,7}$, Georgia Lockwood Estrin ${ }^{8}$, Teodora Gliga ${ }^{9}$, Sheffali Gulati $^{10}$, Mark H. Johnson ${ }^{11}$, Debarati Mukherjee ${ }^{3}$, Vikram Patel ${ }^{12}$, Teressa Tavassoli ${ }^{6}$.

Affiliations: (1) Com DEALL Trust, Bangalore, India, (2) Division of Psychology, Nottingham Trent University, Nottingham, United Kingdom, (3) Centre for Chronic Conditions and Injuries, Public Health Foundation of India, Gurgaon, India, (4) Sangath, New Delhi, India, (5) Computer Science and Engineering, Indian Institute of Technology Bombay, Mumbai, India, (6) Centre for Autism, School of Psychology \& Clinical Language Sciences, University of Reading, Reading, United Kingdom, (7) Division of Psychology, De Montfort University, Leicester, United Kingdom, (8) Centre for Brain and Cognitive Development, Birkbeck University of London, London, United Kingdom, (9) Psychology, University of East Anglia, Norwich, United Kingdom, (10) Child Neurology Division Department of Pediatrics, All India Institute of Medical Sciences, New Delhi, India, (11) Department of Psychology, University of Cambridge, Cambridge, United Kingdom, (12) Department of Global Health and Population, Harvard Medical School, Boston, Massachusetts, United States of America.

Disclosure of potential conflicts of interest: The authors declare that they have no conflict of interest.

Author Contributions: BC, ID, LR designed the tasks: ID and SB collected the data: ID, RB, and SC analyzed the data; ID and BC drafted the manuscript and prepared all figures. The manuscript was reviewed and edited by all the authors. 


\section{References}

1. Srofe LA, Waters $E$. The ontogenesis of smiling and laughter: A perspective on the organization of development in infancy. Psychol Rev. 1976;83: 173-189.

2. Valenza E, Simion F, Cassia VM, Umiltà C. Face preference at birth. J Exp Psychol Hum Percept Perform. 1996;22: 892-903.

3. Bardi L, Regolin L, Simion F. Biological motion preference in humans at birth: role of dynamic and configural properties. Dev Sci. 2011;14: 353-359.

4. Vernetti A, Senju A, Charman T, Johnson MH, Gliga T. Simulating interaction: Using gazecontingent eye-tracking to measure the reward value of social signals in toddlers with and without autism. Dev Cogn Neurosci. 2018;29: 21-29.

5. Höher J, Bach T, Münster A, Bouillon B, Tiling T. Does the Mode of Data Collection Change Results in a Subjective Knee Score? Am J Sports Med. 1997;25: 642-647.

6. Cook C. Mode of administration bias. J Man Manip Ther. 2010;18: 61-63.

7. Kroes G, Veerman JW, De Bruyn EEJ. Bias in Parental Reports? Eur J Psychol Assess. 2003;19: 195-203.

8. Fantz RL. Pattern Vision in Newborn Infants. Science. 1963;140: 296-297.

9. Shimojo S, Simion C, Shimojo E, Scheier C. Gaze bias both reflects and influences preference. Nat Neurosci. 2003;6: 1317-1322.

10. Myowa-Yamakoshi M, Tomonaga M, Tanaka M, Matsuzawa T. Preference for human direct gaze in infant chimpanzees (Pan troglodytes). Cognition. 2003;89: 113-124.

11. Shimojo S, Simion C, Changizi MA. Gaze and Preference-Orienting Behavior as a Somatic Precursor of Preference Decision. The Science of Social Vision. Oxford University Press; 2010. pp. 151-163.

12. Jones W, Carr K, Klin A. Absence of Preferential Looking to the Eyes of Approaching Adults Predicts Level of Social Disability in 2-Year-Old Toddlers With Autism Spectrum Disorder. Arch Gen Psychiatry. 2008;65: 946.

13. Pierce K, Conant D, Hazin R, Stoner R, Desmond J. Preference for geometric patterns early in life as a risk factor for autism. Arch Gen Psychiatry. 2011;68: 101-109.

14. Chakrabarti B, Haffey A, Canzano L, Taylor CP, McSorley E. Individual differences in responsivity to social rewards: Insights from two eye-tracking tasks. Lappe M, editor. PLoS One. 2017;12: e0185146.

15. Gliga T, Elsabbagh $\mathrm{M}$, Andravizou A, Johnson $\mathrm{MH}$. Faces attract infants' attention in complex displays. Infancy. 2009;14: 550-562.

16. Gluckman M, Johnson SP. Attentional capture by social stimuli in young infants. Front Psychol. 2013;4: 527.

17. Spreckelmeyer KN, Krach S, Kohls G, Rademacher L, Irmak A, Konrad K, et al. Anticipation of monetary and social reward differently activates mesolimbic brain structures in men and women. Soc Cogn Affect Neurosci. 2009;4: 158-165.

18. Rademacher L, Salama A, Gründer G, Spreckelmeyer KN. Differential patterns of nucleus accumbens activation during anticipation of monetary and social reward in young and older adults. Soc Cogn Affect Neurosci. 2014;9: 825-831.

19. Dubey I, Ropar D, Hamilton A. Measuring the value of social engagement in adults with and without autism. Mol Autism. 2015;6: 35.

20. Ewing L, Pellicano E, Rhodes G. Using effort to measure reward value of faces in children with autism. PLoS One. 2013;8: e79493.

21. Dubey I, Ropar D, Hamilton A. Brief Report: A Comparison of the Preference for Viewing Social and Non-social Movies in Typical and Autistic Adolescents. J Autism Dev Disord. 2017;47: 514-519.

22. Dubey I, Ropar D, Hamilton AF de C. Social seeking declines in young adolescents. Royal Society Open Science. 2017;4: 170029. 
23. Ruta L, Famà FI, Bernava GM, Leonardi E, Tartarisco G, Falzone A, et al. Reduced preference for social rewards in a novel tablet based task in young children with Autism Spectrum Disorders. Sci Rep. 2017;7: 3329.

24. MRC GCRF Project. [cited 28 Apr 2020]. Available: https://startproject.bhismalab.org/home

25. Pierce K, Marinero S, Hazin R, McKenna B, Barnes CC, Malige A. Eye Tracking Reveals Abnormal Visual Preference for Geometric Images as an Early Biomarker of an Autism Spectrum Disorder Subtype Associated With Increased Symptom Severity. Biol Psychiatry. 2016;79: 657-666.

26. Blatter K, Schultz W. Rewarding properties of visual stimuli. Exp Brain Res. 2006;168: 541-546.

27. Dunn, Dunn, Whetton, Burley. British Picture Vocabulary Scale - Third Edition (BPVS-3). 3rd ed. GL assessment; 1997.

28. Liddle EB, Batty MJ, Goodman R. The Social Aptitudes Scale: an initial validation. Soc Psychiatry Psychiatr Epidemiol. 2009;44: 508-513.

29. Chevallier C, Kohls G, Troiani V, Brodkin ES, Schultz RT. The social motivation theory of autism. Trends Cogn Sci. 2012;16: 231-239.

30. Neuhaus E, Webb SJ, Bernier RA. Linking social motivation with social skill: The role of emotion dysregulation in autism spectrum disorder. Dev Psychopathol. 2019; 1-13.

31. Krafka K, Khosla A, Kellnhofer P, Kannan H, Bhandarkar S, Matusik W, et al. Eye Tracking for Everyone. 2016 IEEE Conference on Computer Vision and Pattern Recognition (CVPR). 2016. doi:10.1109/cvpr.2016.239

32. Bishain R. An Open-Source, Computing Platform-Agnostic, Calibration-Free Preferential Gaze Detection Approach for Social Preference Assessment. INSAR 2019 Annual Meeting. IMFAR; 2019. Available: https://insar.confex.com/insar/2019/webprogram/Paper31575.html

33. Chita-Tegmark M. Social attention in ASD: A review and meta-analysis of eye-tracking studies. Res Dev Disabil. 2016;48: 79-93.

34. Di Giorgio E, Turati C, Altoè G, Simion F. Face detection in complex visual displays: an eye-tracking study with 3- and 6-month-old infants and adults. J Exp Child Psychol. 2012;113: 66-77.

35. Moore A, Wozniak M, Yousef A, Barnes CC, Cha D, Courchesne E, et al. The geometric preference subtype in ASD: identifying a consistent, early-emerging phenomenon through eye tracking. Mol Autism. 2018;9: 19.

36. Shi L, Zhou Y, Ou J, Gong J, Wang S, Cui X, et al. Different Visual Preference Patterns in Response to Simple and Complex Dynamic Social Stimuli in Preschool-Aged Children with Autism Spectrum Disorders. Urgesi C, editor. PLoS One. 2015;10: e0122280.

37. Alexander GM, Wilcox T, Woods R. Sex differences in infants' visual interest in toys. Arch Sex Behav. 2009;38: 427-433.

38. Proverbio AM, Zani A, Adorni R. Neural markers of a greater female responsiveness to social stimuli. BMC Neurosci. 2008;9: 56.

39. Berridge KC, Robinson TE, Aldridge JW. Dissecting components of reward: "liking", "wanting", and learning. Curr Opin Pharmacol. 2009;9: 65-73.

40. Kohls G, Chevallier C, Troiani V, Schultz RT. Social "wanting" dysfunction in autism: neurobiological underpinnings and treatment implications. J Neurodev Disord. 2012;4: 10.

41. Krajbich I, Armel C, Rangel A. Visual fixations and the computation and comparison of value in simple choice. Nat Neurosci. 2010;13: 1292-1298.

42. Kringelbach ML, Berridge KC. Neuroscience of Reward, Motivation, and Drive. Recent Developments in Neuroscience Research on Human Motivation. pp. 23-35.

43. Pool E, Delplanque S, Coppin G, Sander D. Is comfort food really comforting? Mechanisms underlying stress-induced eating. Food Res Int. 2015;76: 207-215.

44. Tibboel H, De Houwer J, Van Bockstaele B. Implicit measures of "wanting" and "liking" in 
humans. Neurosci Biobehav Rev. 2015;57: 350-364.

45. Chevallier C, Grèzes J, Molesworth C, Berthoz S, Happé F. Brief report: Selective social anhedonia in high functioning autism. J Autism Dev Disord. 2012;42: 1504-1509.

46. Da Fonseca D, Santos A, Rosset D, Deruelle C. The "beauty is good" for children with autism spectrum disorders too. Res Autism Spectr Disord. 2011;5: 299-304.

47. Dubey I, Brett S, Ruta L, Bishain R, Chandran S, Bhavnani S, et al. Social is not always special: Evidence for task specificity in measuring social preference in young children. 2019. doi:10.31234/osf.io/3un5e 